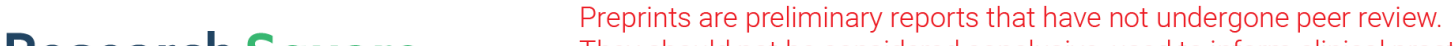 They should not be considered conclusive, used to inform clinical practice, or referenced by the media as validated information. \\ Transport Efficiency of AtGTR1 Dependents on the Hydrophobicity of Transported Glucosinolates
}

\section{Yi-Chia Chung}

Department of Biotechnology and Bioindustry Sciences, National Cheng Kung University

\section{Hao-Yu Cheng}

Department of Biotechnology and Bioindustry Sciences, National Cheng Kung University

\section{Wei-Tung Wang}

Department of Biotechnology and Bioindustry Sciences, National Cheng Kung University

\section{Yen-Jui Chang}

Department of Biotechnology and Bioindustry Sciences, National Cheng Kung University

Shih-Ming Lin ( $\nabla$ smlin@mail.ncku.edu.tw)

Department of Biotechnology and Bioindustry Sciences, National Cheng Kung University

\section{Research Article}

Keywords: Glucosinolates, herbivores, proton, AtGTR1, salicylic acid, phosphomimetic mutation

Posted Date: October 8th, 2021

DOl: https://doi.org/10.21203/rs.3.rs-954528/v1

License: (c) (1) This work is licensed under a Creative Commons Attribution 4.0 International License. Read Full License

Version of Record: A version of this preprint was published at Scientific Reports on March 24th, 2022. See the published version at https://doi.org/10.1038/s41598-022-09115-x. 
Transport Efficiency of AtGTR1 Dependents on the Hydrophobicity of Transported Glucosinolates

Yi-Chia Chung ${ }^{1}$, Hao-Yu Cheng ${ }^{1}$, Wei-Tung Wang ${ }^{1}$, Yen-Jui Chang ${ }^{1}$, Shih-Ming Lin ${ }^{1,2^{*}}$

${ }^{1}$ Department of Biotechnology and Bioindustry Sciences, National Cheng Kung University

${ }^{2}$ Institute of Tropical Plant Sciences and Microbiology, National Cheng Kung University

*Correspondence:smlin@mail.ncku.edu.tw 


\begin{abstract}
Glucosinolates (GLSs) are a group of secondary metabolites that are involved in the defense of herbivores. In Arabidopsis thaliana, Glucosinolate Transporter 1 (AtGTR1) transports GLSs with high affinity via a proton gradient-driven process. In addition to transporting GLSs, AtGTR1 also transports phytohormones, jasmonic acidisoleucine (JA-Ile), and gibberellin (GA). However, little is known about the mechanisms underlying the broad substrate specificity of AtGTR1. Here, we characterized the substrate preference of AtGTR1 by using a yeast uptake assay, and the results revealed that GLS transport rates are negatively correlated with the hydrophobicity of substrates. Interestingly, the AtGTR1 showed a higher substrate affinity for GLSs with higher hydrophobicity, suggesting a hydrophobic substrate binding pocket. In addition, a competition assay revealed that the presence of JA, salicylic acid (SA), and indole-3-acetic acid (IAA) inhibits the transport of GLSs in yeast, suggesting a potential regulatory mechanism of AtGTR1. To further characterize the functional properties of AtGTR1, mutagenesis experiments confirmed that the conserved EXXEK motif and Arg166 are essential for the GLS transport function. In addition, the purified AtGTR1 adopts a homodimeric conformation, which is possibly regulated by phosphorylation on Thr105. The phosphomimetic mutation, T105D, reduced its protein expression and completely abrogated its GLS transport function, indicating the essential role of phosphorylation on AtGTR1. In summary, this study investigated various factors associated with the GLS transport and increased our knowledge on the substrate preferences of AtGTR1. These findings contribute to understanding how the distribution of defense GLSs is regulated in plants and could be used to improve crop quality in agriculture.
\end{abstract}




\section{Introduction}

Nitrate transporter 1/Peptide transporter family (NPF) proteins are proton-coupled symporters that share sequence homologies with Proton-dependent Oligopeptide Transporter (POTs) in animals and bacteria ${ }^{1-3}$. Unlike POTs, which primarily transport di- and tri-peptides, NPF proteins were initially identified as being involved in the regulation of nitrate uptake ${ }^{1,3}$. In recent years, several studies have shown that NPF proteins are also capable of transporting a variety of different substrates, such as auxin (AUX), abscisic acid (ABA), jasmonic acid (JA), gibberellins (GA), monoterpene, and glucosinolates (GLSs) ${ }^{1,4-8}$. These phytochemicals play essential roles in the regulation of plant growth and development, suggesting the importance of NPF proteins in plant physiology. In Arabidopsis thaliana, 53 members of NPF transporters are identified and divided into eight distinct subclades ${ }^{9}$. Among them, AtGTR1 (NPF2.10), a member of NPF2, is known to transport a variety of substrates, including GLSs, nitrate, jasmonic acid-isoleucine (JA-Ile), and GA $\mathrm{GA}^{4,8,10,11}$. It is an interesting topic to investigate how AtGTR1 exhibited a broad range of substrate specificity.

AtGTR1 is primarily expressed in the plasma membrane in the mature leaf and is also involved in regulating GLSs levels in seed ${ }^{8,12}$. In addition, AtGTR1 was also detected in companion cells and was found to import GLSs from the apoplast into the phloem for long-distance transport ${ }^{12}$. These GLSs are a group of secondary metabolites which function in herbivore defense for plants ${ }^{13,14}$. GLSs can be classified into three types based on their precursors. The aliphatic GLSs are primarily derived from methionine, whereas the indole and aromatic GLSs are synthesized from tryptophan and phenylalanine, respectively ${ }^{15}$. AtGTR1-knockout plants showed an unbalanced distribution of all three types of GLSs, resulting in several developmental defects ${ }^{8,10,16}$. In vitro studies have revealed that AtGTR1 transports both aliphatic and indolic GLSs when expressed in Xenopus oocytes ${ }^{2}$. In addition, recent studies using cotton cells expressing AtGTR1 have also shown that AtGTR1 transports the aromatic sinalbin as well as the aliphatic Sinigrin ${ }^{17}$. The molecular structures, sizes, and polarities of the three types of GLS are diverse, and the features between GLSs, JA-Ile, and GA are even more different. The critical molecular signatures of these substrates recognized by AtGTR1 are still not clear, and the mechanism underlying the broad selectivity remains to be elucidated.

The structural study of NPF6.3 (NRT1.1), a dual-affinity nitrate transporter with 28.7\% identity to AtGTR1, has provided important information about the nitrate transport $^{18,19}$. The crystal structure showed that NPF6.3 forms a homodimer, and each monomer is bound with a nitrate ion in the central cavity ${ }^{18,19}$. The dimeric NPF6.3 is known to be dissociated into monomers by phosphorylation of Thr101, and the dissociated NPF6.3 monomer has a high binding affinity for nitrate ions ${ }^{18-20}$. This 
phosphorylation site is also conserved as Thr105 in AtGTR1, and phosphorylation on Thr 105 was found to disrupt its protein dimerization and alter its membrane localization in Xenopus oocytes ${ }^{21}$. However, it is not clear whether the dissociation of AtGTR1 affects the substrate affinity or other transport functions. In addition, another functional motif located on transmembrane helix 1 (TMH 1), EXXEK/R, is also conserved in AtGTR1 (Fig. S1). Jørgensen et al. (2015) have studied each residue of this motif in AtGTR2 and confirmed its functional role in proton translocation and active transport ${ }^{22}$. Although these conserved properties assist in understanding the regulatory and transporting mechanisms of AtGTR1, current studies have mainly revealed the binding sites of nitrate ions or dipeptides and therefore provide little information about the selective mechanisms for transporting GLSs and phytohormones.

To understand how plant cells specifically regulate the transport of these diverse GLSs, we aimed to identify the key factors affecting substrate selectivity of AtGTR1. Here, we expressed AtGTR1 in Saccharomyces cerevisiae and established a yeast uptake assay to measure the GLSs transport activities and study the substrate specificity of AtGTR1. Interestingly, we found that AtGTR1 transports all three types of GLSs and that the GLS transport rates are negatively correlated with substrate hydrophobicity. In addition, the GLSs transport activity was significantly inhibited by the addition of jasmonic acid (JA), indole-3-acetic acid (IAA), and salicylic acid (SA). Overall, this work contributes to increasing our understanding of the substrate preferences of AtGTR1 and provides a new expression system for the characterization of GLS transporters. 


\section{Results}

\section{AtGTR1 was localized in the plasma membrane with GLS transport activity in S. cerevisiae}

To study the transport properties of AtGTR1, we first aimed to establish a heterologous expression system in the yeast $S$. cerevisiae. AtGTR1 fused with a cterminal GFP was constructed to monitor the protein expression and the targeting location of AtGTR1. Fig. 1a shows that AtGTR1-GFP was expressed and primarily localized at the perimeter of the yeast cells with a ring-shaped fluorescence distribution, indicating that AtGTR1 might be localized in the plasma membrane. To further confirm that AtGTR1-GFP expressed in yeast cells are membrane-localized proteins, the yeast membrane fractions were isolated and analyzed by western blot analysis with anti-Histag antibodies and in-gel fluorescence analysis detecting GFP signals (Fig. 1b-d). The results showed that AtGTR1-GFP was present in the yeast membrane fraction with an apparent molecular weight of $75 \mathrm{kDa}$, which was slightly smaller than the theoretical size of $97 \mathrm{kDa}$ (Fig. 1c, d). In addition, a minor band with a molecular weight larger than $180 \mathrm{kDa}$ was detected in both western blot and in-gel fluorescence images (Fig. 1c, d), suggesting that AtGTR1-GFP may form an oligomeric conformation that is not entirely denatured in SDS-PAGE gels. Similarly, when we expressed AtGTR1 without a GFP tag in S. cerevisiae, AtGTR1 was detected in the yeast membrane fractions via western blot as above. Two major bands with apparent molecular sizes of $55 \mathrm{kDa}$ and $140 \mathrm{kDa}$ were observed (Fig. 1b, c), indicating AtGTR1 is also successfully expressed in yeast membranes and might form oligomers as AtGTR1-GFP does. Incidentally, the apparent size of AtGTR1 was also smaller than the theoretical size of $68 \mathrm{kDa}$. Similar gel shifting of membrane proteins in SDS-PAGE gels, caused by SDS-protein interactions and protein conformations, has been reported in the previous studies ${ }^{23}$. Thus, we concluded that both AtGTR1-GFP and AtGTR1 were expressed in yeast and primarily localized to the yeast cell membrane.

After confirming AtGTR1 expression and localization, we established a yeast uptake assay to measure the GLS transport activity and investigate the substrate specificities of AtGTR1. In this assay, yeast cells expressing AtGTR1 and vector control were incubated with GLS for different times, and then intracellular GLS was enriched by solid-state extraction and desulfurized for the following quantification by RP-HPLC-DAD. LC of the control yeast cells showed a clean background without detectable GLSs (Fig. 2a, middle panel), indicating that $S$. cerevisiae does not have a high level of endogenous GLSs or GLS transporters. In contrast, GLSs substantially accumulated in yeast cells expressing AtGTR1 (Fig. 2a, bottom panel). Time-course experiments indicated that the GLS content in the AtGTR1-expressing cells increased 
exponentially and reached saturation after $30 \mathrm{~min}$, whereas the GLS level in the vector control remained non-detectable at all time points (Fig. 2b).

Next, since AtGTR1 is a symporter driven by a proton gradient, the environmental $\mathrm{pH}$ should affect its GLS transport activity. Although previous studies have compared the difference in transport activity of AtGTR1 at $\mathrm{pH} 5$ and $\mathrm{pH} 6{ }^{17}$, a more detailed $\mathrm{pH}$ profiling analysis is still unknown. Thus, we measured the GLS transport activity of AtGTR1 at six different $\mathrm{pH}$ values $(3.5-8.5)$ by using the yeast uptake assay mentioned above. The results showed that the highest GLS transport rate occurred when the $\mathrm{pH}$ of the medium was between 5.5 and 6.5, and the transport activity dropped sharply when the environmental $\mathrm{pH}$ increased from 6.5 to 7.5 (Fig. 2c). In addition, the GLS transport rate also rapidly decreased when the $\mathrm{pH}$ fell below 4.5. These results suggest that AtGTR1 expressed in S. cerevisiae retains its functionality and that the yeast cell uptake assay can be utilized to measure GLS transport properties.

\section{AtGTR1 transports multiple types of GLSs at different rates}

GLSs have the same sulfonated oxime group and $\beta$-thioglucose group, as well as a variable aglycone side chain whose variations are based on different amino acid precursors (Fig. 3a) ${ }^{13,14}$. To understand the effect of various aglycone side chains on AtGTR1 transporting GLS, we selected seven GLSs, including four aliphatic GLSs, two aromatic GLSs, and one indolic GLSs, for measuring AtGTR1 transport activity. These seven GLSs are sinigrin (SIN, aliphatic), gluconapin (GNA, aliphatic), glucoerucin (GER, aliphatic), glucoraphanin (GRA, aliphatic), sinalbin (SNB, aromatic), glucotropaeolin (GTR, aromatic), and glucobrassicin (GBS, indolic), most of which are relatively abundant in Brassica plants (Fig. 3a) ${ }^{24}$. These GLSs were incubated with the yeast cells expressing AtGTR1 for one hour at a final concentration of $100 \mu \mathrm{M}$. The intracellular GLS contents were quantified by RP-HPLC to determine the transport rates. AtGTR1 showed varying transport rates for the uptake of these substrates but did not seem to favor any specific GLS class (Fig. 3b). Among the seven GLSs used in this study, AtGTR1 transported GRA at the highest rate, $439.9 \pm 36.01$ $\mathrm{pmol} / 10^{7}$ cells $/ \mathrm{h}$, followed by SIN at $431.7 \pm 49.09 \mathrm{pmol} / 10^{7} \mathrm{cells} / \mathrm{h}, \mathrm{SNB}$ at $406.2 \pm$ $28.95 \mathrm{pmol} / 10^{7} \mathrm{cells} / \mathrm{h}$, GNA at $347.2 \pm 9.20 \mathrm{pmol} / 10^{7} \mathrm{cell} / \mathrm{s} / \mathrm{h}, \mathrm{GTR}$ at $244.0 \pm 61.2$ $\mathrm{pmol} / 10^{7} \mathrm{cells} / \mathrm{h}$, GER at $198.0 \pm 22.55 \mathrm{pmol} / 10^{7} \mathrm{cells} / \mathrm{h}$, and GBS at $198.2 \pm 13.48$ $\mathrm{pmol} / 10^{7}$ cells $/ \mathrm{h}$ (mean $\pm \mathrm{SD}, \mathrm{n}=3$ ). Interestingly, we found that the transport rates of AtGTR1 for these GLSs was negatively correlated with their retention time during RPHPLC analysis (Fig. 3c); a plot of the substrate accumulation rate and retention time showed that they shared a linear and inverse relationship, with an $\mathrm{R}$ squared value of 0.90 (Fig. 3d). Since the retention time of RP-HPLC correlates with the hydrophobicity 
of the analytes ${ }^{25}$, these results suggest that the transport efficiency of AtGTR 1 is determined by the hydrophobicity of GLSs.

Furthermore, the kinetics of AtGTR1 transporting different GLSs was assessed, and $\mathrm{K}_{\mathrm{m}}$ and $\mathrm{V}_{\max }$ values were calculated to understand the enzymatic properties. We selected three GLSs for kinetic analysis, including aliphatic GRA, aromatic GTR, and indolic GBS. The GLS transport rate of AtGTR1 was measured using yeast uptake assay in the substrate concentration range of $2.5-320 \mu \mathrm{M}$ and the time of substrate transport were changed to 15 minutes to obtain a more accurate initial rate. Fig. 4 revealed that AtGTR1 showed Michaelis-Menten kinetics for transporting all GLSs in yeast. The $\mathrm{K}_{\mathrm{m}}$ values of AtGTR1 are $249.2 \pm 47.23 \mu \mathrm{M}$ for GRA, $13.6 \pm 1.55 \mu \mathrm{M}$ for GTR, and 8.2 $\pm 0.60 \mu \mathrm{M}$ for GBS, whereas the $\mathrm{V}_{\max }$ values are 7,677.6 \pm 810.06 $\mathrm{pmol} / 10^{7}$ cells/h for GRA, $1,536.2 \pm 43.73 \mathrm{pmol} / 10^{7} \mathrm{cells} / \mathrm{h}$ for GTR and 1,509.8 \pm $27.01 \mathrm{pmol} / 10^{7} \mathrm{cells} / \mathrm{h}$ for GBS. These results suggest that AtGTR1 exhibits substrate preference in terms of substrate affinity and transport efficiency.

\section{$J A, I A A$, and $S A$ inhibited the GLS transport activity of AtGTR1 in yeast}

In addition to transporting GLSs, AtGTR1 also transports the phytohormones, JAIle and $\mathrm{GA}^{8,10,11}$. Although these substrates share the commonality of being anions, their molecular structures are quite different from each other. It is still not clear how AtGTR1 recognizes specific phytohormones for transport, and the affinities between these phytohormones and AtGTR1 are also unknown. To answer these questions, five anionic phytohormones, including JA, GA, IAA, ABA, and SA, were selected to compete with GLSs in the yeast uptake assay. Based on the kinetic studies, GTR was chosen for these competition experiments because of its moderate $\mathrm{K}_{\mathrm{m}}$ value compared to GRA and GBS. Moderate substrate affinity of GTR would be helpful to observe the competitive effects of phytohormones with weak affinity. In each experiment, $500 \mu \mathrm{M}$ phytohormones were added against $25 \mu \mathrm{M}$ GTR in the assay medium, and the level of reduction in the GLS transport rate was monitored. As expected, based on the reported affinity of AtGTR1 for JA, the addition of JA significantly reduced the GLS transport efficiency in the yeast uptake assay (Fig. 5a). In contrast, the addition of ABA did not affect the GLS transport significantly (Fig. 5a). Interestingly, although the previous study reports that AtGTR1 can transport GA, the addition of $500 \mu \mathrm{M}$ GA did not reduce GLS transport into yeast cells (Fig. 5a). Unexpectedly, we found that both IAA and SA significantly inhibited GLS transport via AtGTR1 in yeast (Fig. 5a), although neither of these phytohormones has previously been reported as a substrate of AtGTR1.

To further compare the inhibitory efficiencies between JA, IAA, and SA, various concentrations of phytohormones ranging from 62.5 to $2000 \mu \mathrm{M}$ with 2-fold dilution series were added against $25 \mu \mathrm{M}$ GTR in the yeast uptake assay. The reduced GLS 
uptake level was quantified by RP-HPLC and normalized to the control group. The dose-response curves were fitted by nonlinear regression, and the results showed that the GLS transport activity decreased exponentially as the concentration of these phytohormones increased (Fig. 5b-d). The 50\% inhibitory concentration $\left(\mathrm{IC}_{50}\right)$ is 937.8 $\pm 113.85 \mu \mathrm{M}$ for JA, $630.0 \pm 47.51 \mu \mathrm{M}$ for SA and $762.1 \pm 48.14 \mu \mathrm{M}$ for IAA (mean \pm s.e.m., $\mathrm{n}=3$ for each concentration of competitors). Furthermore, both JA and SA showed a Hill slope near -1 (JA: $-0.93 \pm 0.120$; SA: $-1.13 \pm 0.100)$, whereas the Hill slope of IAA is $-1.66 \pm 0.167$, indicating IAA showed a positively cooperative inhibition. Together, these results suggested that JA, IAA, and SA are potential substrates or analogs of AtGTR1.

\section{The GLS transport activity of AtGTR1 requires EXXEK motifs and Arg166 residue}

Previous studies have proposed the substrate-binding mechanisms for transporting nitrate ions and dipeptides in NPF and POT proteins ${ }^{18,19,26}$. However, the specific binding site of GLSs in AtGTR1 have rarely been experimentally confirmed. To understand how AtGTR1 recognizes GLSs, we focused on the key residues located in the central cavity of AtGTR1. Since GLSs are anionic substrates with a sulfonyl group, their negative charge should be balanced by positively charged residues during transport. Moreover, according to the modeling structure, only two positive charged residues, Lys49 and Arg166, are located in the central cavity ${ }^{27}$. Lys49 is part of the conserved EXXEK/R motif on TMH 1, whereas Arg166 is conserved as positively charged residues on the TMH 4 among NPF proteins (Fig. S1). To confirm that these conserved motif and residues are involved in the GLS transport of AtGTR1, we generated four alanine mutants at positions Glu43, Glu48, Lys49, and Arg166 and then observed their GLS transport activity by using yeast uptake assay. The results showed a dramatic decrease in activity for all the mutants (Fig. 6). Western blot analysis confirmed that these mutants were well expressed at the protein level, indicating that the loss of activity was not caused by an absence of expression (Fig. 6). Interestingly, of the residues in the EXXEK motif, the E48A mutant retained a very small amount of GLS transport activity, while the E45A and K49A mutants showed complete loss of function (Fig. 6). In addition, the R166A mutation also completely abrogated the GLS transport function of AtGTR1. These results suggested that the highly conserved EXXEK motif and Arg166 are essential for the GLS transport function of AtGTR1.

\section{AtGTR1 purified from the yeast membrane adopts a dimeric conformation}

Previous studies showed that protein dimerization regulates the nitrate affinity of NPF $6.3^{18,20}$. AtGTR1 was also known to form dimers on the plasma membrane of Xenopus oocytes ${ }^{21}$. Therefore, we want to determine the protein conformation of 
AtGTR1 expressed in S. cerevisiae to explain the GLS transport activity and selectivity observed in this study. As mentioned earlier, when analyzing the yeast membrane of AtGTR1, western blot showed a high molecular weight band, presumably a dimer of AtGTR1 (Fig. 1). To further confirm the dimerization of AtGTR1, we solubilized and purified AtGTR1 from yeast membranes by using immobilized metal affinity chromatography (IMAC), and the purified proteins were subjected to size-exclusion chromatography (SEC) to evaluate the size of the native protein complex. The major peak in the SEC chromatogram was observed at a retention volume corresponding to a molecular weight of $158 \mathrm{kDa}$, which is similar to the theoretical dimeric size of 136 $\mathrm{kDa}$ (Fig. 7a). In addition, a minor shoulder peak, corresponding to a molecular weight of $75 \mathrm{kDa}$, eluted next to the major peak (Fig. 7a). These purified AtGTR1 proteins were analyzed by SDS-PAGE and western blot with anti-His-tag antibody, and the results show a major band at $50 \mathrm{kDa}$ and a minor band at $150 \mathrm{kDa}$ in both IMAC and SEC purified products (Fig. 7b and 7c). Together, the results of SEC and western blot analysis showed that the majority of the AtGTR1 purified proteins adopted a dimeric conformation.

\section{Phosphomimetic mutation T105D reduced the protein stability of AtGTR1 and disrupted its GLS transport}

It was known that phosphorylation of Thr101 of NPF6.3 suppressed the protein dimerization and converted the binding affinity of nitrate to high affinity ${ }^{18,20}$. This threonine residue is conserved in many NPF members, including AtGTR1 (Fig. S1) ${ }^{20}$. To determine whether AtGTR1 is also regulated by phosphorylation at this threonine residue, $\mathrm{T} 105 \mathrm{~A}$ and $\mathrm{T} 105 \mathrm{D}$ mutants were established to mimic constitutively dephosphorylated and constitutively phosphorylated AtGTR1, respectively. These mutants were then used to assay their GLS transport activity. Comparison of the GLS transport activities showed that transport function was completely lost in the T105D mutant, while the T105A mutant showed transport activity similar to that of WT AtGTR1 (Fig. 8a). To further examine whether the decreased activity of the two mutants was related to protein expression levels, the yeast membranes of both mutants were isolated and analyzed by western blot with anti-His-tag antibodies (Fig. 8b). We found that the T105D mutant was expressed at lower levels than the WT and T105A mutant proteins, indicating the decrease in transport activity might be due to the reduced protein expression/stability (Fig. 8b). In addition, a degradation band close to $30 \mathrm{kDa}$ was observed in the T105D membrane but not in the WT and T105A mutants, suggesting that protein stability may be influenced by T105 phosphorylation. These results indicate that Thr105 phosphorylation of AtGTR1 drastically affects its protein properties on the yeast membrane, leading to the loss of GLS transport function. 


\section{Discussion}

Through expressing AtGTR1 in S. cerevisiae, we measured the GLS transport activity of AtGTR1 using a yeast uptake assay coupled with quantification by RPHPLC in this study. Interestingly, AtGTR1 transported all three classes of GLSs, and the GLS transport rates were negatively correlated to the hydrophobicity of substrates. Using a competition assay, we found that the addition of JA, SA, and IAA could inhibit the GLS transport activity of AtGTR1, indicating that these essential phytohormones may be substrates or analogs of AtGTR1. This information on substrate preference provided clues to understand the substrate selective mechanism of AtGTR1.

For characterizing AtGTR1, the yeast GLS uptake assay was utilized to study the $\mathrm{pH}$ profile of AtGTR1. The $\mathrm{pH}$ profiling showed that the optimal $\mathrm{pH}$ for GLS transport is 5.5-6.5, which is similar to the $\mathrm{pH}$ of apoplast, the location of GLS uptake by AtGTR1 in $A$. thaliana ${ }^{12}$. Furthermore, since the cytosolic $\mathrm{pH}$ of yeast is $6.8^{28}$, the poor transport activity observed at $\mathrm{pH} 7.5$ and 8.5 confirmed that proton gradient is essential for AtGTR1 function. These results show the feasibility of this yeast uptake assay for studying GLS transport of AtGTR1. We then utilized this assay to investigate the substrate preference of AtGTR1 and found that the hydrophobicity of GLSs determined the transport rate of AtGTR1 (Fig. 3a). Kinetic analysis showed that AtGTR1 has higher $\mathrm{V}_{\max }$ values for transporting GLSs with lower hydrophobicity, such as GRA. Interestingly, the $\mathrm{K}_{\mathrm{m}}$ value for transporting GRA is higher than transporting GTR and GBS. Therefore, we concluded that AtGTR1 showed a higher substrate affinity to bind the GLSs with higher hydrophobicity, and the tight binding of GLS leads to a lower translocation rate. These results showed that hydrophobicity of the aglycone side chains might decide the substrate preference of AtGTR1. Thus, the hydrophobic interactions between GLSs and AtGTR1 may play an important role in the broad substrate specificity. This hypothesis is supported by the fact that the crystal structure of NPF6.3 shows a hydrophobic cavity for binding nitrate ions ${ }^{18}$. Furthermore, Jørgensen et al. (2017) have proposed that three hydrophobic residues in the central cavity, I52, I53, and L56, may contribute to determining the substrate specificity of $\mathrm{GLSs}^{2}$. Together, the interaction between the aglycone side chains of GLSs and the hydrophobic pockets of AtGTR1 may determine the transport rate of these GLSs.

Furthermore, we conducted the phytohormone competition experiments and found that treatment with JA, SA, and IAA inhibited AtGTR1 from transporting GLS, whereas treatment with ABA or GA did not. It is expected that JA could compete with GLS due to that JA and JA-Ile have been reported as the substrates of AtGTR ${ }^{11}$. Surprisingly, SA treatment also inhibited GLS transport in yeast. Although SA is not reported to be transported via any NPF transporter, including AtGTR1, the inhibitory curve showed the IC50 of SA even lower than JA, suggesting SA may also bind to 
AtGTR1. SA and JA are both phytohormones involved in the regulation of plant immune responses ${ }^{29}$. Interestingly, the application of JA and SA to Arabidopsis and Brassica has been shown to increase the accumulation of $\mathrm{GLSs}^{30-32}$. Furthermore, recent studies showed that SA treatment stimulated the gene expression of GTR1 in Chinese kale ${ }^{33}$. These studies showed the possibility of interactions between SA and AtGTR1. Similarly, IAA showed an inhibitory effect on GLS transport of AtGTR1. IAA has been reported as a substrate for NPF6.3 transport $^{34}$, but there is no evidence of AtGTR1 having IAA transport activity. The $\mathrm{IC}_{50}$ of IAA is slightly higher than that of SA, suggesting that IAA, like SA, may bind to AtGTR1 with a low affinity. The inhibition curve of IAA showed a Hill slop below -1, suggesting a cooperative inhibit the effect of IAA on GLS transport. These results showed that IAA might not only compete for the substrate-binding site with GLSs but also has other effects influence with GLS transport. On the other hand, despite being a known substrate of AtGTR1, GA does not affect GLS transport. This may be since AtGTR1 transports GA at a slower rate than it transports GLS ${ }^{10,21}$. Besides, it is also possible that AtGTR1 transports GA through another pathway, different from the GLS. These competition experiments revealed that JA, IAA, and SA might serve as substrates and/or analogs of AtGTR1. More evidence from biochemical and physiological studies is needed to clarify the functional roles of these phytohormones on AtGTR1 transport activity.

In addition to exploring the substrate preference of AtGTR1, we focused on studying the functional residues in the central cavity of AtGTR1. The mutagenesis studies confirmed that four charged residues, Glu45, Glu48, Lys49, and Arg166, are essential for GLS transport (Fig. 5a). Interestingly, in our study, the E48A mutant retained a very small amount of GLS transport activity, whereas E45A and K49A lost GLS transport function completely (Fig. 5a). The residual activity of E48A suggested that Glu48 may play a different role from Glu45 in the proton coupling mechanism. Outside of this motif, another mutant, R166A, completely lost its GLS transport function. This positively charged residue on $\mathrm{TMH} 4$ has been proposed to interact with a dipeptide substrate in POT protein ${ }^{3,35}$. In this work, the loss of function of the R166A mutant supports that Arg166 may participate in capturing GLSs during the transport cycle. These mutagenesis experiments suggested that the proton coupling and substrate binding mechanisms of AtGTR1 are similar to other NPF proteins.

Moreover, it is known that protein conformation is essential for regulating the function of membrane transporters ${ }^{36}$. For example, the lactose transporter, LacS, forms a dimer with cooperative activities ${ }^{37}$; and the dual-affinity nitrate transporter, NPF6.3, showed high nitrate affinity as a monomer while forming a homodimer with low affinity $^{20}$. Here, we revealed that the AtGTR1 purified from $S$. cerevisiae adopts a homodimeric conformation. However, the functional significance of the AtGTR1 
dimerization remains unclear because the decoupled AtGTR1 has not been functionally characterized. Unlike that phosphorylation of NPF6.3 induced protein dissociation ${ }^{20}$, phosphorylation of AtGTR1 caused protein degradation at yeast membrane (Fig. 8b). A recent study has also reported that phosphorylation in AtGTR1 regulates the plasma membrane localization in Xenopus oocytes ${ }^{21}$. Thus, we speculate that phosphorylation of AtGTR1 may lead to dimer dissociation and further result in reduced stability. Nevertheless, it remains to be confirmed whether this phosphorylation of AtGTR1 occurs in plants. Proteomic analysis of the post-translational modifications on AtGTR1 in Arabidopsis would be helpful to understand the regulation of AtGTR1 signaling.

This study showed several interesting characteristics of the yeast-expressed AtGTR1 and found that JA, IAA, and SA significantly inhibited the GLS transport function of AtGTR1 in S. cerevisiae. However, it is still unclear how these phytohormones interact with AtGTR1, and thus the mechanism of broad selectivity of AtGTR1 remains unknown. Previous studies have shown that AtGTR1 is involved in phytohormone signaling and physiological regulation ${ }^{10,11,38}$. Thus, it is crucial to elucidate the phytohormone transport mechanisms of AtGTR1. Protein structure studies will be helpful to reveal this unknown mechanism. Solving the structure of AtGTR1 binding to phytohormone molecules will provide solid evidence to elucidate the molecular mechanism. In this study, we have established the purification protocols to isolate the AtGTR1 proteins from the yeast membrane (Fig. 7b). These protocols could be further utilized to prepare purified AtGTR1 for protein chrysography and cryo-EM studies.

Additionally, the phytohormones that can inhibit AtGTR1 transporting GLS have the potential to act as growth regulators to control GLS levels in crops. A recent study showed that knockout of GTR1 orthologs reduced seed GLS contents in Brassica rapa and Brassica juncea ${ }^{39}$. Therefore, we hypothesize that the treatment of inhibitors and analogs of GTR1 may also reduce the GLS contents in plants. These results increased our knowledge of the plant transporters regulating the distribution of defense metabolites and contributed to the development of modern agriculture. 


\section{Material \& Methods}

\section{Plasmid construction and mutagenesis}

cDNA encoding AtGTR1 with a C-terminal 8-his-tag was synthesized and inserted into the pESC-URA vector (Agilent Technologies, U.S.A.) between the restriction sites, BamHI and XhoI. To express AtGTR1-GFP, AtGTR1 cDNA was subcloned into the pDDGFP-2 vector (a kind gift from Dr. David Drew at Stockholm University) between the restriction sites, BamHI and XmaI. All the mutants used in this study were generated by using the In-Fusion ${ }^{\circledR}$ HD Cloning Kit (Takara Bio, U.S.A.) and confirmed by DNA sequencing. The PCR primer pairs used to generate mutants are listed in Supplementary Table S1.

\section{Yeast transformation and expression}

The plasmid constructs were transformed into the $S$. cerevisiae strain FGY217 (MATa, ura3-52, lys2 $\Delta 201$, and pep4 $\Delta$; a kind gift from Dr. David Drew at Stockholm University) by the LiAc/polyethylene glycol method ${ }^{40}$. The yeast transformants were cultured in complete minimal $(\mathrm{CM})$ medium $(1.7 \%(\mathrm{w} / \mathrm{v})$ yeast nitrogen base, $5 \%(\mathrm{w} / \mathrm{v})$ ammonium sulfate, $2 \%(\mathrm{w} / \mathrm{v})$ glucose, $0.01 \%(\mathrm{w} / \mathrm{v}) \mathrm{L}-\mathrm{lysine} ; \mathrm{pH} 5.5)$ at $30^{\circ} \mathrm{C}$ for $18 \mathrm{~h}$. Some of the overnight cultures were stored at $-80^{\circ} \mathrm{C}$ with the addition of $15 \%$ glycerol, while the rest were diluted in fresh $\mathrm{CM}$ medium and cultured at $30^{\circ} \mathrm{C}$ until the $\mathrm{OD}_{600}$ reached 1.0. To induce protein expression of AtGTR1, the medium was replaced with CM-galactose containing $2 \%(\mathrm{w} / \mathrm{v})$ galactose instead of glucose, and the yeast cells were induced at $30^{\circ} \mathrm{C}$ for $48 \mathrm{~h}$.

\section{Yeast cell uptake assay and phytohormone competition assay}

Yeast cells expressing AtGTR1 were washed twice and resuspended in a fresh CM medium containing $20 \mathrm{mM}$ sodium citrate at $\mathrm{pH}$ 5.5. The cell density was determined and adjusted to $1 \times 10^{8}$ cells $/ \mathrm{ml}$ based on $\mathrm{OD}_{600}$ values. One milliliter of the cells was then harvested and resuspended in $1 \mathrm{ml}$ of $\mathrm{CM}$ medium containing $20 \mathrm{mM}$ sodium citrate and $100 \mu \mathrm{M}$ GLSs. The uptake of GLSs by yeast cells was carried out at $30^{\circ} \mathrm{C}$ for $1 \mathrm{~h}$ and terminated by centrifugation of the yeast cells at $6,000 \mathrm{xg}$ for $5 \mathrm{~min}$. The harvested yeast cells were immediately lysed for subsequent GLS quantification as described in the next section. For $\mathrm{K}_{\mathrm{m}} / \mathrm{V}_{\max }$ determination, GRA, GTR, and GBS were selected as the substrate and diluted to 8 concentrations $(2.5,5,10,20,40,80,160$, and $320 \mu \mathrm{M})$ in the assay medium. The uptake rate was measured at an incubation time of 15 min. 
In the phytohormone competition experiments, one milliliter of $1 \times 10^{8}$ cells $/ \mathrm{ml}$ yeast cells expressing AtGTR1 were harvested and resuspended in $1 \mathrm{ml} \mathrm{CM}$ medium containing $20 \mathrm{mM}$ sodium citrate, $25 \mu \mathrm{M}$ GTR, and $500 \mu \mathrm{M}$ of each phytohormone. These samples were then incubated at $30^{\circ} \mathrm{C}$ for $1 \mathrm{~h}$ and terminated as mentioned above. After wash by fresh CM medium twice, the yeast cells were harvested by centrifugation and lysed immediately for GLS quantification by HPLC as described in the following section. In addition, to obtaining the competition curves of JA, IAA, and SA, the following concentrations of phytohormones were used to compete with $25 \mu \mathrm{M}$ GTR: 0 , $62.5,125,250,500,1000,2000 \mu \mathrm{M}$. Other experimental steps were the same as the above competition experiments to acquire the transport rate of GTR under different phytohormone concentrations.

\section{GLS quantification by RP-HPLC-DAD}

The GLS quantification procedures were performed according to previous studies with modifications ${ }^{41,42}$. The yeast cells that had taken up the GLSs were lysed in $70 \%$ methanol containing $1 \mu \mathrm{M}$ GNA as the internal standard and then incubated at $75^{\circ} \mathrm{C}$ for $10 \mathrm{~min}$. After centrifugation at 4,000 x g for $10 \mathrm{~min}$ to remove the insoluble precipitates, the supernatant was loaded onto $0.25 \mathrm{ml}$ of DEAE Sephadex resin, which was activated and pre-equilibrated with $70 \%$ methanol. After sample binding, the resin was washed twice with $70 \%$ methanol and distilled water. The resin was then washed with desulfation buffer (20 mM MES, $\mathrm{pH} 5.2$ ), and $0.125 \mathrm{U}$ of sulfatase was added to remove the sulfite group of the GLSs. The resin was then incubated at $25^{\circ} \mathrm{C}$ for $18 \mathrm{~h}$ in the dark. The digested GLSs were eluted from the resin and quantified by using an Agilent 1260 HPLC system with a C18 column (Poroshell 120 EC-C18, 3.0 x $100 \mathrm{~mm}, 2.7 \mu \mathrm{m}$, Agilent Technologies, U.S.A.). The mobile phase consisted of solvent A (distilled water) and solvent B (methanol), with a linear gradient of $1-19 \%$ solvent $B$ over 25 min. The flow rate was $0.8 \mathrm{ml} / \mathrm{min}$, and the column temperature was $30^{\circ} \mathrm{C}$. The $\mathrm{UV}$ absorbance was monitored at $229 \mathrm{~nm}$. The peak area of each GLS was integrated by the Openlab ChemStation program and corrected by using the internal standard peaks. The standard curve of each type of GLS was established by using $0.25,0.5,1,2,4,8$, and $16 \mu \mathrm{M}$ samples.

\section{Yeast microsomal membrane isolation}

The method used to isolate yeast microsomal membranes was carried out as described in previous studies with minor modifications ${ }^{43}$. Yeast cells that expressed AtGTR1 or AtGTR1-GFP were washed with yeast wash buffer (0.1 M Tris-HCl (pH 9.4), $7 \mathrm{mM}$ 2-mercaptoethanol) and then incubated in Zymolyase medium (100 mM Tris-HCl, 1\% (w/v) yeast extract, 2\% (w/v) peptone, 1\% (w/v) glucose, $700 \mathrm{mM}$ 
sorbitol, $5 \mathrm{mM}$ 2-mercaptoethanol, $30 \mathrm{mg} / \mathrm{ml}$ Zymolyase; $\mathrm{pH} 8.0$ ) at $30^{\circ} \mathrm{C}$ for $3 \mathrm{~h}$. The yeast spheroplasts were collected by centrifugation, resuspended in lysis buffer $(50 \mathrm{mM}$ Tris-ascorbate, $5 \mathrm{mM}$ EGTA-Tris, $10 \% \quad(\mathrm{w} / \mathrm{v})$ glycerol, $1.5 \% \quad(\mathrm{w} / \mathrm{v})$ polyvinylpyrrolidone 40,000 (PVP40000), $1 \mathrm{mM}$ pepstatin $\mathrm{A}$, and $1 \mathrm{mM}$ phenylmethanesulfonyl fluoride (PMSF); pH 7.6) and homogenized by ultrasonication on ice. The sonication intensity was limited to $960 \mathrm{~J} / \mathrm{mL}$ using an ultrasonic generator (UP200S, Hielscher, Germany). After removing the cell debrides by centrifugation at $4,000 \mathrm{x}$ g for $10 \mathrm{~min}$, the supernatant was ultracentrifuged at $100,000 \mathrm{x} \mathrm{g}$ for $60 \mathrm{~min}$ at $4^{\circ} \mathrm{C}$. The microsomal membranes in the pellet were resuspended in storage buffer ( 50 $\mathrm{mM}$ Tris-HCl, $400 \mathrm{mM} \mathrm{NaCl}, 20 \%$ (w/v) glycerol; $\mathrm{pH}$ 7.6) and ultracentrifuged again to remove the residual soluble and peripheral membrane proteins. The microsomal membranes were resuspended in storage buffer for subsequent protein purification.

AtGTR1 purification and SEC analysis

The membrane protein solubilization and purification methods were as described in previous studies with optimization for $A t G T R 1^{43}$. The yeast microsomal membrane containing AtGTR1 or AtGTR1-GFP was diluted with storage buffer (50 mM Tris-HCl, $400 \mathrm{mM} \mathrm{NaCl}, 20 \%(\mathrm{w} / \mathrm{v})$ glycerol; $\mathrm{pH}$ 7.6) containing 1\% (w/v) n-Dodecyl $\beta$-Dmaltoside (DDM) to a final protein concentration of $3 \mathrm{mg} / \mathrm{ml}$ and gently stirred at $4^{\circ} \mathrm{C}$ for $60 \mathrm{~min}$. After solubilization, the samples were ultracentrifuged at 100,000 x $\mathrm{g}$ for 1 $\mathrm{h}$ at $4^{\circ} \mathrm{C}$. The supernatant was purified on a Ni-NTA column with an FPLC system (ÄKTA pure, Cytiva, U.S.A.). After washing the column with $50 \mathrm{mM}$ imidazole, AtGTR1 was eluted with IMA buffer (50 mM Tris-HCl, $400 \mathrm{mM} \mathrm{NaCl}, 20 \%$ (w/v) glycerol, $0.03 \%(\mathrm{w} / \mathrm{v}) \mathrm{DDM}, 0.03 \%(\mathrm{w} / \mathrm{v})$ sodium cholate, $1 \mathrm{mM}$ pepstatin A; $\mathrm{pH} 7.6)$ containing $300 \mathrm{mM}$ imidazole. The purified AtGTR1 was dialyzed against SEC buffer (20 mM Tris-HCl, $150 \mathrm{mM} \mathrm{NaCl}, 10 \%$ (w/v) glycerol, 0.03\% (w/v) DDM, $1 \mathrm{mM}$ pepstatin $\mathrm{A} ; \mathrm{pH}$ 7.6) and concentrated for SEC. SEC is also conducted by using an FPLC system, and $1 \mathrm{mg}$ of purified AtGTR1 was injected into a Superdex S200 pg $10 / 600$ column and eluted with SEC buffer at $4{ }^{\circ} \mathrm{C}$. The fractions containing dimeric AtGTR1 were collected and concentrated for further analysis.

\section{Statistical analysis}

Significant differences between two groups were assessed by a two-tailed unpaired Student t-test. Multiple group comparisons were performed by one-way ANOVA followed by Tukey's multiple comparisons test. The $\mathrm{K}_{\mathrm{m}}$ and $\mathrm{V}_{\max }$ values and associated standard errors were obtained by nonlinear regression estimation to fit the MichaelisMenten equation. The IC50 value was calculated by using nonlinear regression 
estimation to fit the [inhibitor] versus normalized response equation with variable Hill slope; no curve fitting restraints were included. 


\section{References}

1. Wang, Y. Y., Cheng, Y. H., Chen, K. E. \& Tsay, Y. F. Nitrate transport, signaling, and use efficiency. Annu. Rev. Plant Biol. 69, 85-122 (2018).

2. Jørgensen, M. E. et al. Origin and evolution of transporter substrate specificity within the NPF family. Elife 6, 1-31 (2017).

3. Prabhala, B. K., Rahman, M., Nour-eldin, H. H., Jørgensen, F. S. \& Mirza, O. PTR2/POT/NPF transporters: what makes them tick? Advances in Protein Chemistry and Structural Biology 123, (Elsevier Ltd, 2021).

4. Chiba, Y. et al. Identification of Arabidopsis thaliana NRT1/PTR FAMILY (NPF) proteins capable of transporting plant hormones. J. Plant Res. 128, 679686 (2015).

5. Tal, I. et al. The Arabidopsis NPF3 protein is a GA transporter. Nat. Commun. 7, (2016).

6. Kanno, Y. et al. Identification of an abscisic acid transporter by functional screening using the receptor complex as a sensor. Proc. Natl. Acad. Sci. U. S. A. 109, 9653-9658 (2012).

7. Payne, R. M. E. et al. An NPF transporter exports a central monoterpene indole alkaloid intermediate from the vacuole. Nat. Plants 3, 16208 (2017).

8. Nour-Eldin, H. H. et al. NRT/PTR transporters are essential for translocation of glucosinolate defence compounds to seeds. Nature 488, 531-534 (2012).

9. Léran, S. et al. A unified nomenclature of nitrate transporter 1/peptide transporter family members in plants. Trends Plant Sci. 19, 5-9 (2014).

10. Saito, H. et al. The jasmonate-responsive GTR1 transporter is required for gibberellin-mediated stamen development in Arabidopsis. Nat. Commun. 6, 111 (2015).

11. Ishimaru, Y. et al. GTR1 is a jasmonic acid and jasmonoyl-L-isoleucine transporter in Arabidopsis thaliana. Biosci. Biotechnol. Biochem. 81, 249-255 (2017).

12. Andersen, T. G. et al. Integration of biosynthesis and long-distance transport establish organ-specific glucosinolate profiles in vegetative arabidopsis. Plant Cell 25, 3133-3145 (2013).

13. War, A. R. et al. Mechanisms of plant defense against insect herbivores. Plant Signal. Behav. 7, 1306-1320 (2012).

14. Reichelt, M. et al. Benzoic acid glucosinolate esters and other glucosinolates from Arabidopsis thaliana. Phytochemistry 59, 663-671 (2002).

15. Ishida, M., Hara, M., Fukino, N., Kakizaki, T. \& Morimitsu, Y. Glucosinolate metabolism, functionality and breeding for the improvement of brassicaceae vegetables. Breed. Sci. 64, 48-59 (2014). 
16. Xu, D. et al. GTR-Mediated Radial Import Directs Accumulation of Defensive Glucosinolates to sulfur-Rich cells in the phloem cap of Arabidopsis inflorescence stem. Mol. Plant 12, 1474-1484 (2019).

17. Nambiar, D. M., Kumari, J., Arya, G. C., Singh, A. K. \& Bisht, N. C. A cell suspension based uptake method to study high affinity glucosinolate transporters. Plant Methods 16, 1-13 (2020).

18. Sun, J. et al. Crystal structure of the plant dual-affinity nitrate transporter NRT1.1. Nature 507, 73-77 (2014).

19. Parker, J. L. \& Newstead, S. Molecular basis of nitrate uptake by the plant nitrate transporter NRT1.1. Nature 507, 68-72 (2014).

20. Liu, K.-H. \& Tsay, Y.-F. Switching between the two action modes of the dualaffinity nitrate transporter CHL1 by phosphorylation. EMBO J. 22, 1005-1013 (2003).

21. Ishimaru, Y. et al. Dimerization of GTR1 regulates their plasma membrane localization. Plant Signal. Behav. 12, 1-4 (2017).

22. Jørgensen, M. E. et al. A functional EXXEK motif is essential for proton coupling and active glucosinolate transport by NPF2.11. Plant Cell Physiol. 56, 2340-2350 (2015).

23. Rath, A., Glibowicka, M., Nadeau, V. G., Chen, G. \& Deber, C. M. Detergent binding explains anomalous SDS-PAGE migration of membrane proteins. Proc. Natl. Acad. Sci. U. S. A. 106, 1760-1765 (2009).

24. Bhandari, S. R. et al. Profiling of individual desulfo-glucosinolate content in cabbage head (Brassica oleracea var. Capitata) germplasm. Molecules 25, 1-15 (2020).

25. Valkó, K. Chromatographic hydrophobicity index by fast-gradient RP-HPLC: A high-throughput alternative to $\log \mathrm{P} / \log$ D. Anal. Chem. 69, 2022-2029 (1997).

26. Longo, A., Miles, N. W. \& Dickstein, R. Genome mining of plant NPFs reveals varying conservation of signature motifs associated with the mechanism of transport. Front. Plant Sci. 871, 1-17 (2018).

27. Jørgensen, M. E. et al. Origin and evolution of transporter substrate specificity within the NPF family. Elife 6, (2017).

28. Van Eunen, K. et al. Measuring enzyme activities under standardized in vivolike conditions for systems biology. FEBS J. 277, 749-760 (2010).

29. Robert-Seilaniantz, A., Grant, M. \& Jones, J. D. G. Hormone crosstalk in plant disease and defense: more than just jasmonate-salicylate antagonism. Annu. Rev. Phytopathol. 49, 317-343 (2011). 
30. Kiddle, G. A., Doughty, K. J. \& Wallsgrove, R. M. Salicylic acid-induced accumulation of glucosinolates in oilseed rape (Brassica napus L.) leaves. J. Exp. Bot. 45, 1343-1346 (1994).

31. Ku, K. M., Jeffery, E. H. \& Juvik, J. A. Exogenous methyl jasmonate treatment increases glucosinolate biosynthesis and quinone reductase activity in kale leaf tissue. PLoS One 9, (2014).

32. Doughty, K. J., Kiddle, G. A., Pye, B. J., Wallsgrove, R. M. \& Pickett, J. A. Selective induction of glucosinolates in oilseed rape leaves by methyl jasmonate. Phytochemistry 38, 347-350 (1995).

33. Jiang, D. et al. Molecular cloning and characterization of three glucosinolate transporter (GTR) genes from Chinese kale. Genes (Basel). 10, (2019).

34. Krouk, G. et al. Nitrate-regulated auxin transport by NRT1.1 defines a mechanism for nutrient sensing in plants. Dev. Cell 18, 927-937 (2010).

35. Léran, S. et al. Functional characterization of the Arabidopsis abscisic acid transporters NPF4.5 and NPF4.6 in Xenopus oocytes. Front. Plant Sci. 11, 1-6 (2020).

36. Alguel, Y., Cameron, A. D., Diallinas, G. \& Byrne, B. Transporter oligomerization: Form and function. Biochem. Soc. Trans. 44, 1737-1744 (2016).

37. Veenhoff, L. M., Heuberger, E. H. M. L. \& Poolman, B. The lactose transport protein is a cooperative dimer with two sugar translocation pathways. EMBO J. 20, 3056-3062 (2001).

38. Kuo, H. Y., Kang, F. C. \& Wang, Y. Y. Glucosinolate Transporter1 involves in salt-induced jasmonate signaling and alleviates the repression of lateral root growth by salt in Arabidopsis. Plant Sci. 297, 110487 (2020).

39. Nour-Eldin, H. H. et al. Reduction of antinutritional glucosinolates in Brassica oilseeds by mutation of genes encoding transporters. Nat. Biotechnol. 35, 377382 (2017).

40. Gietz, R. D., Schiestl, R. H., Willems, A. R. \& Woods, R. A. Studies on the transformation of intact yeast cells by the LiAc/SS-DNA/PEG procedure. Yeast 11, 355-360 (1995).

41. Madsen, S. R., Olsen, C. E., Nour-Eldin, H. H. \& Ann Halkier, B. Elucidating the role of transport processes in leaf glucosinolate distribution. Plant Physiol. 166, 1450-1462 (2014).

42. Hogge, L. R., Reed, D. W., Underhill, E. W. \& Haughn, G. W. HPLC separation of glucosinolates from leaves and seeds of Arabidopsis thaliana and their identification using thermospray liquid chramatography/mass spectrometry. $J$. Chromatogr. Sci. 26, 551-556 (1988). 
43. Lin, S. M. et al. Crystal structure of a membrane-embedded $\mathrm{H}^{+}$-translocating pyrophosphatase. Nature 484, 399-403 (2012). 


\section{ACKNOWLEDGMENTS}

We would like to thank Dr. David Drew at Stockholm University, Sweden, for kindly providing the plasmids and yeast strains used to complete this study. The authors gratefully acknowledge the use of EM000900 of MOST 110-2731-M-006-001 belonging to the Core Facility Center of National Cheng Kung University. The authors would like to acknowledge funding from the Ministry of Science and Technology (MOST) of Taiwan (MOST 109-2636-B-006 -012; MOST 110-2636-B-006 -012) to SML.

\section{AUTHOR CONTRIBUTIONS}

YCC performed the experiments, made Figs. 2,3, and 5-8, made the supplementary figures, wrote $1 / 3$ of the manuscript draft, and edited the manuscript. HYC made Fig. 1 , assisted in performing the yeast uptake assay, established the HPLC analysis methods, and edited the manuscript. WTW assisted with the kinetic experiments and prepared Fig. 4. YJC constructed the plasmids, assisted with the reference list, and edited the manuscript. SML acquired funding, designed the experiments, wrote the manuscript, and provided advice and technical guidance. 


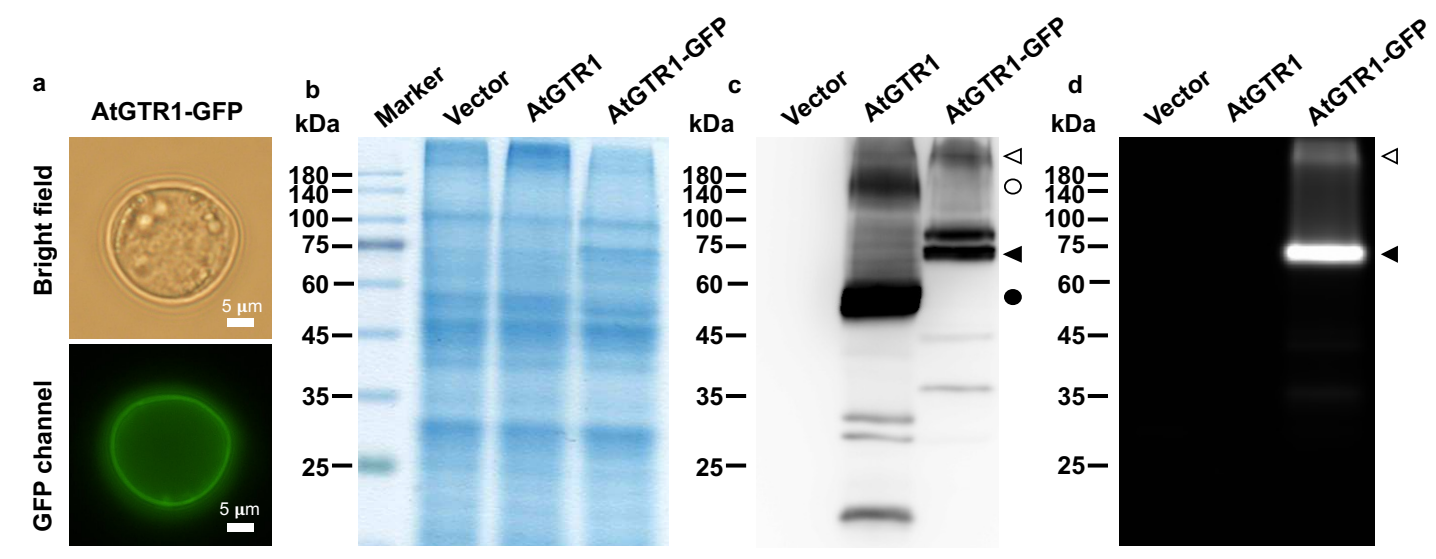

Figure 1. Heterologously expressed AtGTR1 localizes to the yeast membrane. (a) Microscopic observation of AtGTR1-GFP-expressing yeast cells. The scale bar represents $5 \mu \mathrm{m}$. (b, c, d) The yeast membrane fractions isolated from AtGTR1-GFPexpressing and AtGTR1-expressing yeasts were analyzed by (b) SDS-PAGE, (c) western blotting, and (d) in-gel fluorescence. The primary antibody is an anti-His-tag antibody. The filled triangle and filled circle indicate the predicted positions of the AtGTR1-GFP and AtGTR1 bands, respectively. The open triangle and open circle indicate the predicted positions of dimeric AtGTR1-GFP and AtGTR1 bands, respectively. 

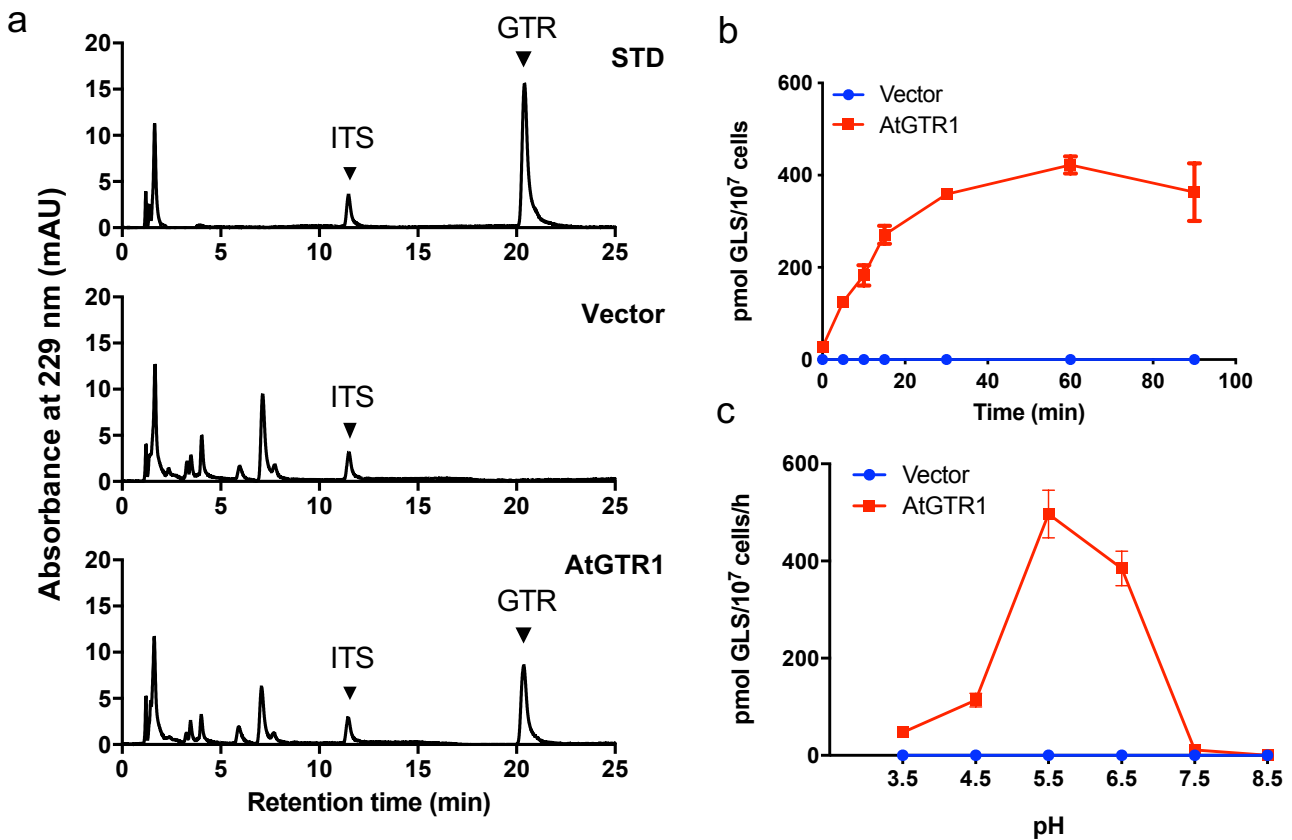

Figure 2. Yeast uptake assay showed the GLS transport properties of AtGTR1. (a) RP-HPLC analysis for measuring GLS transport rates in yeast uptake assay. Upper panel: $4 \mu \mathrm{M}$ glucotropaeolin (GTR) as the standard (STD); Middle and Bottom panel: yeast cells expressing vector and AtGTR1 were incubated with $100 \mu \mathrm{M}$ GTR for 1h, respectively. All samples were spiked with $1 \mu \mathrm{M}$ gluconapin (GNA) as the internal control (ITS) and then treated with solid-state extraction and desulfation for RP-HPLC quantification. (b) Time-dependent GLS accumulation via AtGTR1. Yeast cells expressing AtGTR1 were incubated with $100 \mu \mathrm{M}$ GTR for different lengths of time, and then GLS contents of the yeasts were quantified by RP-HPLC. (c) pH profile of the GLS transport of AtGTR1. Yeast cells were incubated in the assay medium containing $100 \mu \mathrm{M}$ GTR with various $\mathrm{pH}$ for $1 \mathrm{~h}$ and then lysed to quantify the GLS contents by RP-HPLC. All data points with error bars represented the mean \pm SD of independent three repeats. 


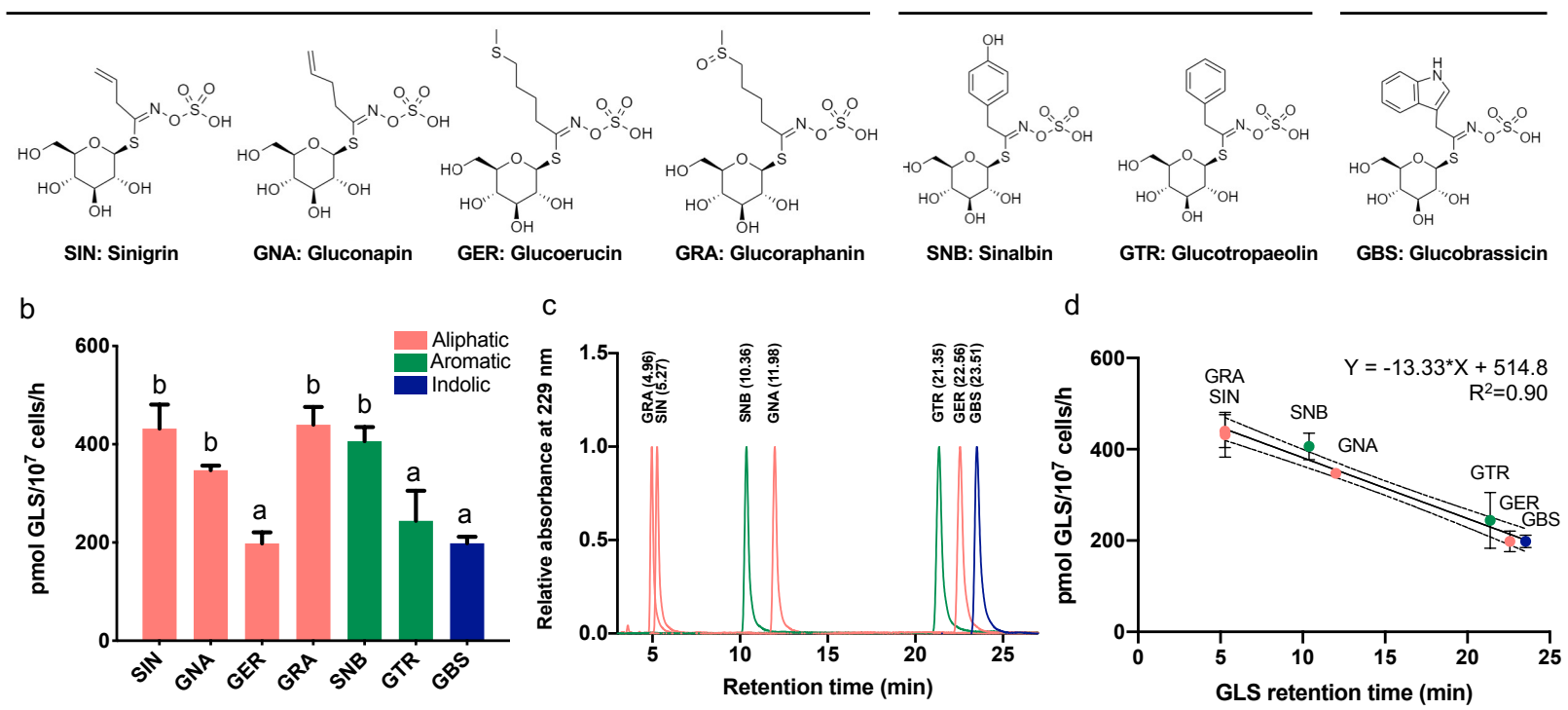

Figure 3. Substrate preference of AtGTR1 depends on the hydrophobicity of transported GLSs. (a) Molecular structures of the seven GLSs used in this study. The corresponding type is marked at the top. (b) Transport activity of AtGTR1 for the seven tested GLSs as measured by yeast uptake assay. The GLS transported in yeast cells were quantified by RP-HPLC. Letters above bars indicate statistically significant differences between groups (one-way ANOVA, $p<0.05$ ). Bar values are the means of three independent replicates. Error bars indicate the SD. (c) RP-HPLC chromatogram of each desulfo-GLS standard. The compound name and retention time are marked at the top of the peaks. (d) The transport activity of each GLS was plotted against its retention time. The linear correlation function is shown, and the dashed line shows the confidence interval of $95 \%$. Each data point represents mean $\pm \operatorname{SD}(n=3)$. The pink color represents aliphatic GLSs, the green color represents aromatic GLSs, and the blue color represents indolic GLSs. 

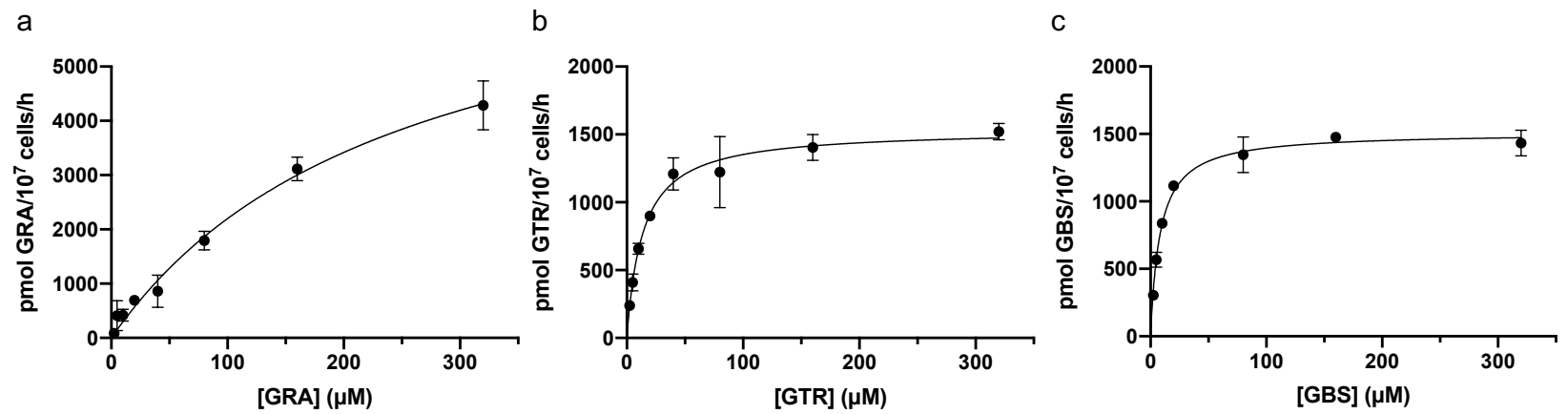

Figure 4. AtGTR1 showed high affinity and low efficiency for transporting GTR and GBS. The kinetic curve of AtGTR1 transporting (a) GRA (b) GTR, and (c) GBS was measured by yeast uptake assay in the substrate concentration range of 2.5-320 $\mu \mathrm{M}$. Michaelis-Menten kinetic curves were fitted using nonlinear regression, and the apparent $\mathrm{K}_{\mathrm{m}}$ for GRA, GTR, and GBS are 249.2, 13.6, and $8.2 \mu \mathrm{M}$, respectively. Each data point represents the mean of three independent replicates (mean $\pm \mathrm{SD}$ ). 
a



b
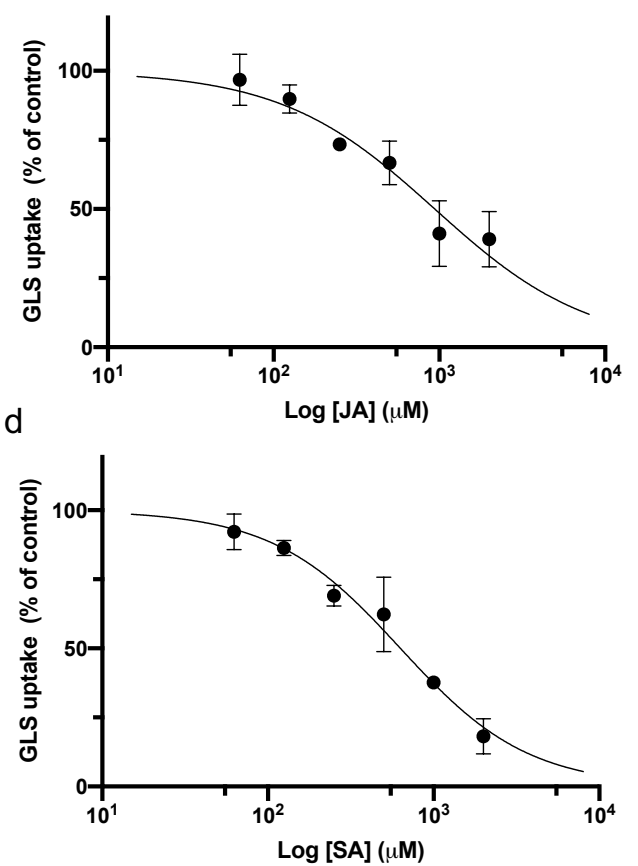

Figure 5. The addition of JA, IAA, and SA inhibited the transport of GLS via AtGTR1. (a) Yeast GLS uptake activity was evaluated in the presence of various acidic phytohormones. In each experiment, yeast cells expressing AtGTR1 were incubated with $25 \mu \mathrm{M}$ GLS and $500 \mu \mathrm{M}$ phytohormones for $1 \mathrm{~h}$. GLS contents in the yeast cells were quantified by RP-HPLC and were normalized to the average value of the control group. Ctl: control group, without adding phytohormones; JA: jasmonic acid; GA: gibberellic acid; SA: salicylic acid; IAA: indole-3-acetic acid; and ABA: abscisic acid. Bar values are the means of three independent replicates. Error bars indicate SD. Significant differences were assessed by unpaired two-tailed Student t-test and are indicated by ${ }^{* *} p<0.01$. (b-d) Concentration-response curves for the inhibition of GLS uptake by addition of (b) JA, (c) IAA, and (d) SA. Various concentrations of the phytohormones were treated to inhibit GLS transport in yeast uptake assay. The GLS contents in yeast cells were quantified and normalized to the mean of the control group. Concentration-response curves were fitted using nonlinear regression. Corresponding $\mathrm{IC}_{50}$ values for JA, IAA, and SA are $937.8 \mu \mathrm{M}, 762.1 \mu \mathrm{M}$ and $630.0 \mu \mathrm{M}$, respectively. Each data point represents mean $\pm \operatorname{SD}(n=3)$. 




Figure 6. EXXEK and Art166 are involved in the transport function of AtGTR1. The GLS transport activity of AtGTR1 mutants. The GLS transport activity was determined by yeast uptake assay, and the protein expression of these mutants was confirmed by western blot. The primary antibody is an anti-His-tag antibody. Bar values with error bar represent mean \pm S.D. $(\mathrm{n}=3)$; ${ }^{* *} p<0.01$; N.D. indicates nondetectable. 

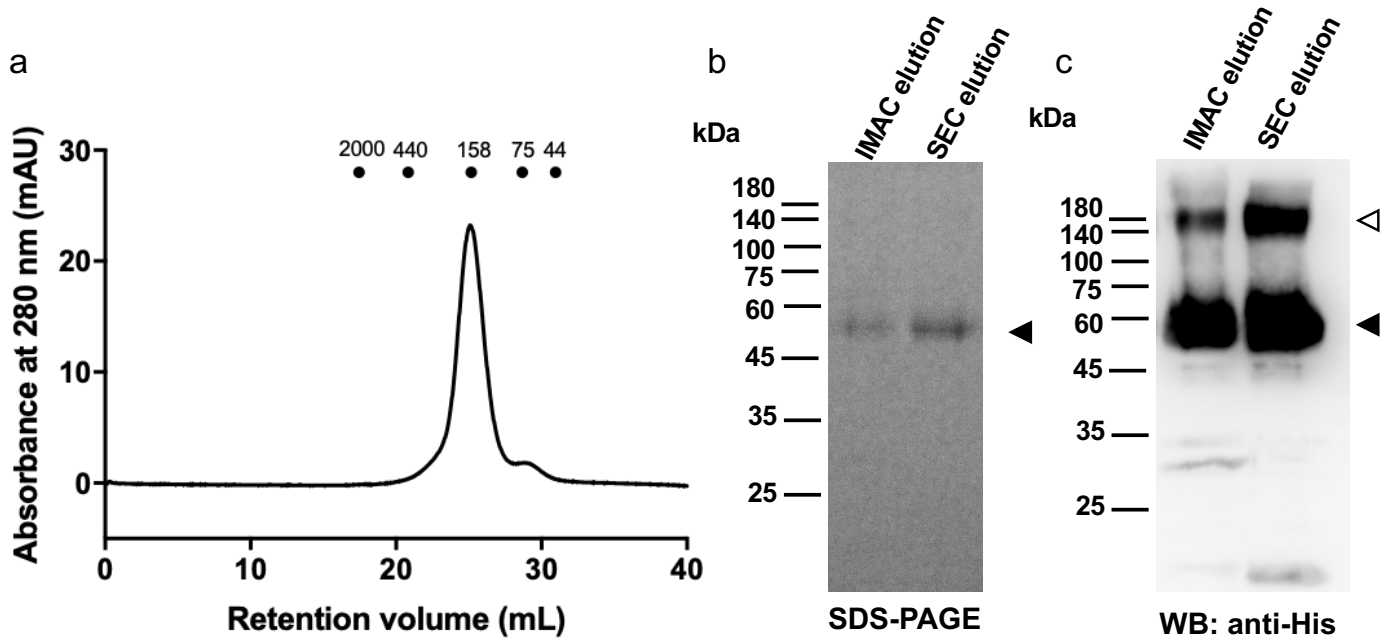

Figure 7. Purified AtGTR1 primarily adopts a homodimeric conformation. (a) SEC chromatogram of AtGTR1 showed that the major eluted peak corresponded to molecular size near $158 \mathrm{kDa}$. Points indicate the retention volume of each standard protein marker with labeled molecular sizes $(\mathrm{kDa})$. (b-c) The AtGTR1 purified by IMAC and SEC were respectively analyzed by (b) SDS-PAGE and (c) western blot with anti-His tag antibody. The filled triangle indicates the AtGTR1 monomer, whereas the open triangle indicates the predicted dimer. 
a

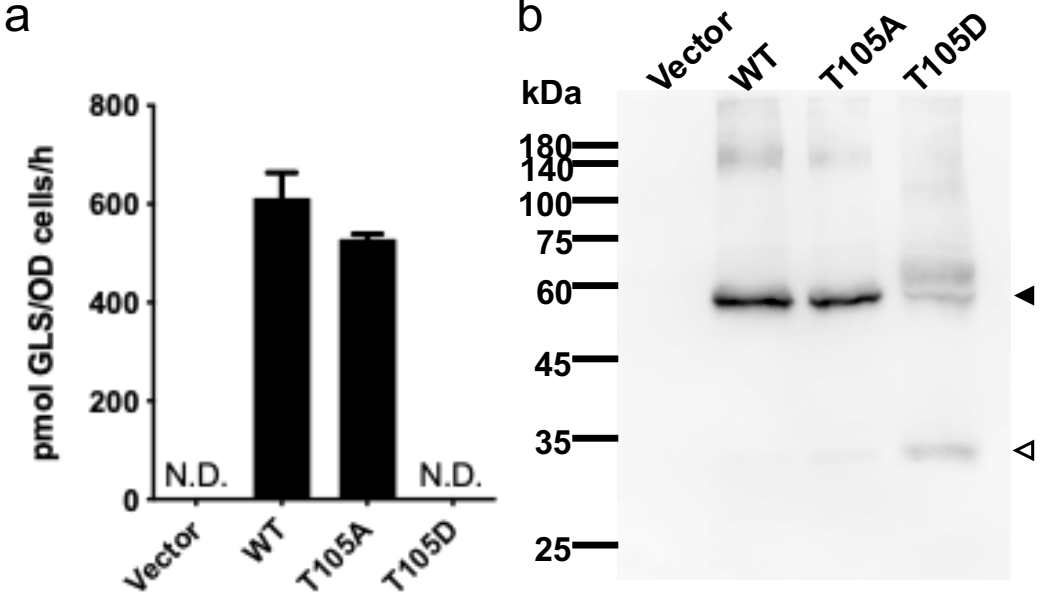

Figure 8. Phosphomimetic mutant of AtGTR1 lost GLS transport function and protein expression in yeast. (a) GLS transport activity of T105 mutants was determined by yeast uptake assay. The GLS uptake was quantified by RP-HPLC and showed as vertical bars with errors (mean $\pm S D, n=3$ ); N.D. indicates nondetectable. (b) Western blot of yeast cells expressing AtGTR1 and its T105 mutants. An anti-His-tag antibody was used as the primary antibody. The filled triangle indicates the AtGTR1 monomer, whereas the open triangle indicates degraded AtGTR1. 


\section{Supplementary Files}

This is a list of supplementary files associated with this preprint. Click to download.

- SupplementaryTablesFigures.pdf 